\title{
Electrophysiological course of uraemic neuropathy in haemodialysis patients
}

\author{
T Ogura, A Makinodan, T Kubo, T Hayashida, Y Hirasawa
}

\begin{abstract}
The objective of this study was to confirm electrophysiologically both the presence and course of uraemic neuropathy in haemodialysis patients. Nerve conduction studies of the lower extremities were done in 70 haemodialysis patients and 20 normal volunteers. Compared with that in normal volunteers, the distal motor latency in the tibial nerve of patients was prolonged significantly $(p<0.05)$, and the minimal $F$ wave latency in the tibial nerve was also prolonged significantly $(p<0.05)$. Motor conduction velocity in the tibial nerve was reduced significantly $(p<0.05)$, and sensory nerve conduction velocity in the medial plantar nerve also was reduced significantly $(p<0.05)$. These results suggest the presence of uraemic neuropathy in haemodialysis patients. Twenty patients were investigated by a follow up study five years later. Parameters from $F$ wave conduction studies, which were thought to be the most useful in the evaluation of neuropathy, showed no significant differences between the initial and follow up trials. These observations suggest that uraemic neuropathy does not progress during haemodialysis. These results also suggest that most haemodialysis patients showed electrophysiological evidence of uraemic neuropathy, but no remarkable electrophysiological change in uraemic neuropathy during haemodialysis was recognised.

(Postgrad Med $\mathcal{F}$ 2001;77:451-454)
\end{abstract}

Keywords: uraemic neuropathy; haemodialysis; electrophysiological study

Department of

Orthopaedic Surgery,

Kyoto Prefectural

University of

Medicine, Kamigyo,

Kyoto 602-8566, Japan

T Ogura

A Makinodan

T Kubo

T Hayashida

Y Hirasawa

Correspondence to:

Dr Ogura

togura@koto.kpu-m.ac.jp

Submitted 22 February 2000 Accepted 6 November 2000

Chronic renal failure is known to be complicated by uraemic neuropathy. The typical clinical features of uraemic neuropathy include paresthesias, such as burning sensations in the feet rather than in the upper extremities. ${ }^{1}$ The number of patients exhibiting clinical features of uraemic neuropathy has recently decreased because of the technical improvements in haemodialysis. ${ }^{2}$ It has been pointed out, however, that the incidence of uraemic neuropathy with electrophysiological abnormalities is still high. ${ }^{3}$ There has been no consensus on whether it improves with time..$^{4-7}$ Nerve conduction studies in the

Table 1 Patient characteristics

\begin{tabular}{lllll}
\hline & No of cases & Sex $(M / F)$ & $\begin{array}{l}\text { Mean (SD) } \\
\text { age (years) }\end{array}$ & $\begin{array}{l}\text { Duration (range) of } \\
\text { haemodialysis (years) }\end{array}$ \\
\hline Haemodialysis patients & 70 & $31 / 39$ & $61.4(10.5)$ & $11.1(0.3-28)$ \\
Normal volunteers & 20 & $12 / 8$ & $60.7(10.5)$ & \\
\hline
\end{tabular}

extremities are used to evaluate the severity and progress of uraemic neuropathy. Because patients who have undergone haemodialysis for more than 10 years commonly develop carpal tunnel syndrome, that is, entrapment neuropathy, ${ }^{8-11}$ care should be exercised in interpreting the findings of the nerve conduction studies. The ulnar nerve may be used occasionally to eliminate the influence of carpal tunnel syndrome, and cubital tunnel syndrome has been similarly reported as a complication. ${ }^{9}$ It can hardly be said that the problem of the effect of entrapment neuropathy has been solved. On the other hand, anatomically the compression factors are less along the course of the nerves of the lower extremities, unlike the carpal tunnel, and therefore the nerves of the lower limbs are suitable for the analysis of uraemic neuropathy developing during haemodialysis. In the present study, peripheral nerve dysfunction during dialysis and the chronological changes in dysfunction were evaluated by nerve conduction studies of the lower extremities in patients undergoing haemodialysis. In addition, nerve conduction studies were conducted on the upper extremities, and the results were compared with those from the lower extremities.

\section{Patients and methods}

PATIENTS

The subjects were 70 patients who underwent haemodialysis between 1 December 1992 and 1 December 1997. There were 31 males and 39 females, with an age range of 39-86 years (mean (SD) 61.4 (10.5) years). The duration of haemodialysis ranged from three months to 28 years (mean (SD) 11.1 (3.5) years). The patients were divided into three groups by the duration of haemodialysis as follows: less than 10 years in group 1 (mean (SD) 5.0 (3.0) years, $\mathrm{n}=30$ ); more than 10 years and less than 20 years in group 2 (mean (SD) 13.7 (3.0) years, $\mathrm{n}=29)$; and more than 20 years in group 3 (mean (SD) $22.3(2.6)$ years, $\mathrm{n}=11$ ). Twenty normal volunteers served as controls: 12 males and eight females, with an age range of 31-75 years (mean (SD) 60.7 (11.8) years) (table 1). The present study was carried out according to the ethical regulations of the Helsinki Declaration.

\section{NERVE CONDUCTION STUDIES OF THE LOWER} EXTREMITIES

In the haemodialysis patients and normal subjects, the tibial nerve was examined as the motor nerve, whereas the sural and medial plantar nerves were examined as the sensory nerves. In the tibial nerve, the motor nerve conduction velocity (MCV) and the distal motor latency (DML) were measured by stimulating the popliteal region and the ankle 
Table 2 Comparison of the values in nerve conduction studies between normal subjects and haemodialysis patients

\begin{tabular}{lccl}
\hline & Normal subjects & $\begin{array}{l}\text { Haemodialysis } \\
\text { patients }\end{array}$ & p Value \\
\hline Tibial & $4.9(0.8)$ & $5.3(0.9)$ & $0.03^{\star}$ \\
DML (ms) & $48.1(3.0)$ & $41.6(5.1)$ & $0.001^{\star}$ \\
MCV (m/s) & $41.6(3.0)$ & $39.2(3.8)$ & 0.22 \\
FCV (m/s) & $39.6(3.0)$ & $41.3(3.9)$ & $0.04^{\star}$ \\
F minimal latency (ms) & $45.8(3.8)$ & $44.7(5.0)$ & 0.37 \\
Sural SCV (m/s) & $46.5(4.1)$ & $42.1(5.1)$ & $0.003^{\star}$ \\
Medial plantar SCV (m/s) & &
\end{tabular}

DML: distal motor latency; FCV: F wave conduction velocity; MCV: motor nerve conduction velocity; SCV: sensory nerve conduction velocity. ${ }^{\star} \mathrm{p}<0.05$.

Table 3 Comparison of the values in nerve conduction studies among the three groups with different durations of haemodialysis

\begin{tabular}{lccc}
\hline \multicolumn{3}{c}{ Duration of haemodialysis (years) } \\
\cline { 2 - 4 } & Group 1 (<10) & Group 2 (10-20) & Group 3 (20+) \\
\hline Median & & & \\
DML (ms) & $3.7(0.3)$ & $4.8(2.4)$ & $6.5(2.7)^{\star}$ \\
MCV (m/s) & $52.1(3.8)$ & $49.4(5.5)$ & $46.4(6.4)^{\star}$ \\
SCV (m/s) & $50.9(5.0)$ & $45.8(11.3)$ & $34.8(8.9)^{\star}$ \\
Tibial & & & \\
DML (ms) & $5.2(1.0)$ & $5.5(0.8)$ & $5.5(1.1)$ \\
MCV (m/s) & $41.6(4.0)$ & $42.2(6.4)$ & $39.8(3.7)$ \\
FCV (m/s) & $39.8(3.0)$ & $39.1(4.5)$ & $38.4(3.5)$ \\
F minimal latency (ms) & $40.6(2.6)$ & $42.0(4.7)$ & $41.0(3.5)$ \\
Medial plantar SCV (m/s) & $42.6(4.3)$ & $41.0(5.4)$ & $42.6(6.7)$ \\
Sural SCV (m/s) & $44.9(4.5)$ & $44.1(5.2)$ & $45.5(5.8)$ \\
\hline
\end{tabular}

DML: distal motor latency; FCV: F wave conduction velocity; MCV: motor nerve conduction velocity; SCV: sensory nerve conduction velocity. ${ }^{\star} \mathrm{p}<0.05$.

joint. The compound muscle action potential (CMAP) was recorded with a silver-silver (I) chloride plate electrode placed on the abductor hallucis muscle. The DML was taken as the latency of any change from the baseline CMAP recorded after stimulation of the ankle joint 10 $\mathrm{cm}$ proximal to the recording electrode. The $\mathrm{F}$ wave was obtained by stimulating the popliteal region and recorded with a silver-silver (I) chloride plate electrode placed on the abductor hallucis muscle. Stimulation was applied 16 times at a frequency of $1 \mathrm{~Hz}$. All the trials were recorded, and the minimal latency and $F$ wave conduction velocity were measured. The $\mathrm{F}$ wave conduction velocity was calculated using the following equation: (the distance between the 12 th thoracic vertebral spinous process and the popliteal region $\times 2) /(F$ wave latency $-M$ wave latency-1 ms). ${ }^{12}$ The medial plantar nerve conduction velocity was measured by antidromically stimulating the nerves in the ankle joint proximal to the tarsal tunnel and recording the sensory nerve action potential (SNAP) with a ring electrode placed on the big toe. The stimulation was applied at a frequency of $2 \mathrm{~Hz}$, and 100 trials were averaged. In the sural nerve, the sensory nerve conduction velocity (SCV) was measured by antidromically stimulating the nerves in the calf and recording SNAP with a silver-silver (I) chloride plate electrode placed on the lateral aspect of the ankle joint. Patients were followed up and the measurements were conducted again after an interval of five years.

NERVE CONDUCTION STUDIES OF THE UPPER EXTREMITIES

In haemodialysis patients, DML, MCV, and SCV were measured for the median nerve. The DML was defined as the latency of any change from baseline in the CMAP obtained by placing a silver-silver (I) chloride plate electrode on the abductor pollicis brevis muscle and stimulating the median nerve at the wrist joint $7 \mathrm{~cm}$ proximal to the recording electrode. The median nerve SCV was measured by antidromically stimulating the nerves of the wrist joint and deducting the SNAP recorded from the ring electrode placed on the index finger. The stimulation was applied at a frequency of $2 \mathrm{~Hz}$ and repeated 20 times.

\section{STATISTICAL ANALYSIS}

All values were reported as mean (SD), and a p value less than 0.05 was considered statistically significant. Student's $t$ test was used for testing the significance of differences in the values measured in the nerve conduction studies in the normal subjects and haemodialysis patients. Measured values among the haemodialysis groups, stratified by the duration of haemodialysis, were compared using one way layout analysis of variance, and if significant, the difference in the mean values was tested for significance using the Scheffe F test.

\section{Results}

NERVE CONDUCTION STUDIES OF THE LOWER EXTREMITIES

The tibial nerve CMAP was obtained successfully in all the normal subjects. The sural nerve SNAP was obtained successfully in 18 subjects (90\%): the SCV was $45.8(3.8) \mathrm{m} / \mathrm{s}$, and the amplitude was 14.3 (3.6) $\mu \mathrm{V}$. The medial plantar nerve SNAP was obtained successfully in 17 subjects ( $85 \%)$ : the SCV was $46.5(4.1)$ $\mathrm{m} / \mathrm{s}$ and the amplitude was $1.6(0.8) \mu \mathrm{V}$. In the haemodialysis patients, the tibial nerve CMAP was obtained successfully in all the patients, and the medial plantar nerve SNAP was obtained successfully in 38 patients (54\%). The sural nerve SNAP was obtained successfully in 61 patients $(87 \%)$. The tibial nerve DML, the tibial nerve MCV, the tibial nerve minimal $\mathrm{F}$ wave latency, and the medial plantar nerve SCV in the patient groups were significantly different from corresponding values of the normal subjects $(\mathrm{p}<0.05)$. These results are summarised in table 2 .

In comparing the three groups with different durations of haemodialysis, there were no changes in the tibial nerve DML, the tibial nerve MCV, the values measured by the tibial nerve $\mathrm{F}$ wave conduction study, the sural nerve SCV, and the medial plantar nerve SCV. These results are summarised in table 3.

The follow up study of the same haemodialysis patients revealed a significant prolongation of the tibial nerve DML after five years (6.0 (1.0) $\mathrm{ms}$ ) compared with the initial DML value (5.1 (0.5) ms; $\mathrm{p}<0.05)$. All other parameters were essentially unchanged. The tibial nerve MCV was 40.9 (3.6) $\mathrm{m} / \mathrm{s}$ at the initial measurement and 39.9 (3.0) $\mathrm{m} / \mathrm{s}$ after five years. The $\mathrm{F}$ wave conduction velocity was 38.8 (3.4) $\mathrm{m} / \mathrm{s}$ at the initial measurement and 39.1 (2.4) $\mathrm{m} / \mathrm{s}$ after five years. The tibial nerve minimal $\mathrm{F}$ wave latency was $40.8(2.8) \mathrm{ms}$ at the initial measurement and $42.2(2.4) \mathrm{ms}$ after five years. The medial plantar nerve SCV was 
Table 4 Comparisons of the values in nerve conduction studies between two trials

\begin{tabular}{lccc}
\hline & Initial trial & $\begin{array}{l}\text { Second trial } \\
\text { (5 years later) }\end{array}$ & p Value \\
\hline Tibial & & & \\
DML (ms) & $5.1(0.5)$ & $6.1(1.0)^{\star}$ & $0.001^{\star}$ \\
MCV (m/s) & $40.9(3.6)$ & $39.9(3.0)$ & 0.12 \\
FCV (m/s & $38.8(3.4)$ & $39.1(2.4)$ & 0.59 \\
F minimal latency (ms) & $40.8(2.8)$ & $42.2(2.4)$ & 0.21 \\
Medial plantar SCV (m/s) & $39.0(6.7)$ & $40.2(4.4)$ & 0.61 \\
\hline
\end{tabular}

DML: distal motor latency; FCV: F wave conduction velocity; MCV: motor nerve conduction velocity; SCV: sensory nerve conduction velocity. ${ }^{\star} \mathrm{p}<0.05$.

$39.0(6.7) \mathrm{m} / \mathrm{s}$ at the initial measurement and $40.2(4.4) \mathrm{m} / \mathrm{s}$ after five years (table 4$)$.

NERVE CONDUCTION STUDIES IN THE LOWER AND UPPER EXTREMITIES

A comparison of the values measured by the nerve conduction studies in the lower and upper extremities showed a significant prolongation of the median nerve DML in group 3 compared with that in group $1(\mathrm{p}<0.05)$. In the tibial nerve DML, however, no significant prolongation was observed when the duration of haemodialysis was prolonged. The median nerve SCV was significantly lower in group 3 than in group 1 $(\mathrm{p}<0.05)$. However, the medial plantar nerve SCV was unchanged when the duration of haemodialysis was prolonged. The median nerve MCV was significantly lower in group 3 than in group $1(\mathrm{p}<0.05)$. However, the tibial nerve $\mathrm{MCV}$ was unchanged when the duration of haemodialysis was prolonged (table 3 ).

\section{Discussion}

Peripheral nerve neuropathy can be classified roughly into two pathological types: (1) one mainly due to axonal degeneration, and (2) one mainly due to demyelination. ${ }^{13}$ Electrophysiologically, nerve conduction studies of the four extremities have demonstrated decreased amplitude of the evoked potentials in the former type and decreased conduction velocity in the latter type. The pathological state of uraemic neuropathy is a multiple neuropathy due to axonal degeneration of the sensory and motor nerves starting from the lower extremities with secondary development of demyelination. ${ }^{14}{ }^{15}$ To evaluate the presence or absence of uraemic neuropathy in patients undergoing haemodialysis, we conducted nerve conduction studies of the lower extremities in haemodialysis patients and compared the results with those of normal subjects. The sensory nerve conduction studies showed reduction of the medial plantar nerve conduction velocity in the haemodialysis patients compared with the normal subjects. Recording was impossible in 13\% of the haemodialysis patients for the sural nerve and in $46 \%$ for the medial plantar nerve. However, recording was also impossible in $10 \%$ of the normal subjects for the sural nerve and in $15 \%$ for the medial plantar nerve. The cause of this failure of recording may have been the effect of age ${ }^{16}$ because small amplitude evoked potentials of a sensory nerve are known to be affected by aging. On the other hand, the CMAP and the $\mathrm{F}$ wave of the motor nerves were obtained successfully in all the haemodialysis patients. The tibial nerve MCV decreased compared with that of the normal subjects. However, the tibial nerve DML, which reflects disturbance of the distal peripheral nerve, and the minimal $\mathrm{F}$ wave latency, ${ }^{17}$ which is the most sensitive parameter in detecting slightly delayed conduction observed in peripheral axonal neuropathy, were prolonged. From the electrophysiological results, we predicted a high incidence of uraemic neuropathy in patients undergoing haemodialysis.

The haemodialysis patients were divided into three groups according to the duration of haemodialysis, and the values measured in the nerve conduction studies of the groups were compared. With prolongation of the duration of haemodialysis, the median nerve DML was prolonged, and the MCV and the SCV decreased in the upper extremities. On the other hand, in the lower extremities, there were no apparent changes in the tibial nerve MCV, the sural nerve SCV, and the medial plantar nerve SCV. In reviewing these results, the known increased incidence of carpal tunnel syndrome in haemodialysis patients with prolongation of haemodialysis duration may serve as a good comparative example (31\% to $57 \%$ at more than 10 years, and $71 \%$ at more than 20 years). Because the median nerve evoked potential was obtained from the abductor pollicis muscle and index finger by stimulating the wrist joint, the median nerve DML and SCV might have been affected by median neuropathy because of carpal tunnel syndrome. The forearm motor nerve conduction velocity, which represents the function of the nerves proximal to the carpal tunnel, also decreased. This decrease may be explained by (1) development of retrograde degeneration of the nerve fibres, ${ }^{18}$ and (2) selective injury of the thick fibres ${ }^{19}$ by mechanical compression at the entrapment point. Therefore, the effect of entrapment neuropathy on the median nerve impedes evaluation of the severity of uraemic neuropathy using the upper extremities. The lower extremities allow precise evaluation of the severity of uraemic neuropathy because they rarely develop tarsal tunnel syndrome, ${ }^{20}$ which is an entrapment neuropathy. Our nerve conduction studies of the lower extremities demonstrated the absence of changes in the tibial nerve DML, the tibial nerve MCV, the values measured by the tibial nerve $F$ wave conduction study, the sural nerve SCV, and the medial plantar nerve SCV in the patient groups with different haemodialysis durations. Only the tibial nerve DML was prolonged after five years. The medial plantar nerve SCV, which reflects the most distal peripheral nerve function, was not reduced, and there were no significant changes in the other values measured, including the minimal $F$ wave latency, ${ }^{21}$ which does not accompany reduction in the tibial nerve MCV and is most reproducible among all the nerve conduction studies. Reproducibility of the tibial nerve DML is problematic; its value has shown the most variability among nerve conduction parameters. Even in the nerve conduction studies, the 
measurement values of tibial nerve DML vary greatly, and their reproducibility has been considered troublesome. The minimal $\mathrm{F}$ wave latency was the least variable with a fluctuation rate of $5 \%$, and the tibial nerve MCV showed a fluctuation rate of approximately $10 \%$. However, the tibial nerve DML varied greatly, with a variation rate of $24 \%$, and the low reproducibility has been attributed to insufficient intensity of stimulation of the tibial nerve at the ankle due to some anatomical factors. Many previous reports have described chronological reductions in the nerve conduction velocities during haemodialysis. ${ }^{4-622}$ In the present study, the addition of an F wave conduction study, which showed less fluctuation of the measured values, enabled us to demonstrate that the severity of uraemic neuropathy remains unchanged during haemodialysis.

1 Kanda T. Peripheral neuropathy in uremia. Neurol Med 1987;26:8-17. 2 Thomas PK. Screening for peripheral neuropathy in patients treated

3 Miyazima S. Neuropathy. Kidney and Dialysis 1986;21:5814.

4 Leone M, Bottacchi E, Alloatti S, et al. Follow-up of nerve conduction in chronic uremic patients during hemodialysis. Ital F Neurol Sci 1992;13:317-21.

5 Caccia MR, Mangili A, Mecca G, et al. Effects of hemodialytic treatment on uremic polyneuropathy. A clinical and electrophysiological follow-up study. $\mathcal{F}$ Neurol $1977 ; 217$ : 113-23

6 Bazzi C, Pagani C, Sorgato G, et al. Uremic polyneuropathy: a clinical and electrophysiological study in 135 short- and long-term hemodialyzed patients. Clin Nephrol 1991;35: $176-81$.

7 Jebson RH, Teckhoff H, Honet JC. Natural history of uremic polyneuropathy and effects of dialysis. $N$ Engl $\mathcal{F} \mathrm{Med}$ $1967 ; 277: 327-33$.
8 Warren DJ, Otieno LS. Carpal tunnel syndrome in patients on intermittent haemodialysis. Postgrad Med $\mathcal{f}$ 1975;51:4502

9 Delmez JA, Holtmann B, Sicard GA, et al. Peripheral nerve entrapment syndromes in chronic hemodialysis patients. Nephron 1982;30:118-23.

10 Halter SK, Delisa JA, Stolov WC, et al. Carpal tunnel syndrome in chronic renal dialysis patients. Arch Phys Med Rehabil 1982;62:197-201.

11 Ogura T. A pathophysiological study of carpal tunnel syndrome in patients with long-term hemodialysis. F Kyoto Pref Univ Med 1994;103:1113-28.

12 Kimura J. Electrodiagnosis in diseases of nerve and muscle: principles and practice. Philadelphia: FA Davis Company, 1989: 332-52.

13 Yasuda T, Sohue G. Pathophysiology and origin of a neuropathy. Fournal of Medical Technology 1996;40:760-6.

14 Thomas PK, Hollinrake K, Lascelles RG, et al. The polyneuropathy of chronic renal failure. Brain 1971;94:76180 .

15 Dyck PJ, Johnson WJ, Lambert EH, et al. Segmental demyelination secondary to axonal degeneration in uremic neuropathy. Mayo Clin Proc 1971;46:400-31.

16 Guiloff RJ, Sherratt RM. Sensory conduction in medial plantar nerve: normal values, clinical applications, and a comparison with the sural and upper limb sensory nerve action potentials in peripheral neuropathy. $f$ Neurol action potentials in peripheral neuro
Neurosurg Psychiatry 1977;40:1168-81.

17 Kimura J. Medical technology of neuropathy. Fournal of Medical Technology 1996;40:757-9.

18 Yamano Y, Okunobou Y, Inoue T. Follow up studies of severe carpal tunnel syndrome with reduced conduction velocity of proximal segment. Orthopaedic Surgery and Traumatology 1986;29:1767-73.

19 Saito T, Akagi S, Sasai K, et al. Proximal slowing in entrapment neuropathy. F Jpn Soc Surg Hand 1994;11:381-4.

20 Samoto N, Akiyama T, Nishida T, et al. A case of tarsal tunnel syndrome due to an amyloid tumor in a long-term hemodialysis patient. Orthopaedic Surgery and Traumatology 1992;40:1408-12.

21 Kohara N, Kimura J, Kaji R, et al. Inter-trial variability of nerve conduction studies: multicenter analysis. Fapanese Fournal of Electroencephalography and Electromyography 1994; 22:384-93.

22 Okuda Y, Asai H, Yasui A, et al. Changes of nerve conduction velocity in patients undergoing long-term hemodialysis. F Fpn Soc Surg Hand 1989;6:377-81. 